\title{
The Cbl-b RING finger domain has a limited role in regulating inflammatory cytokine production by IgE-activated mast cells
}

Morten P Oksvold ${ }^{\dagger}$, Samantha A Dagger, Christine B F Thien and Wallace Y Langdon*

School of Surgery and Pathology, University of Western Australia, Crawley, Western Australia 6009, Australia

* Corresponding author.

Tel : 6189346 2939; Fax : 6189346 2891; Email: wlangdon@cyllene.uwa.edu.au

${ }^{\dagger}$ Current address:

Institute of Pathology, Faculty of Medicine, University of Oslo

Rikshospitalet-Radiumhospitalet Medical Centre

N-0027 Oslo, Norway.

Abbreviations:

BMMC, bone marrow-derived mast cell; BR/BR, Cbl-b RING finger mutant mouse; CIN85, Cbl-interacting protein of 85kDa; IKK, IKB kinase; LAT linker for activation of $T$ cells; MCP-1, monocyte chemotactic peptide-1.

Keywords: Mast Cells, Cytokines, Fc Receptors, Allergy, NFkB.

Running title: Negative regulation of mast cell signalling by Cbl-b. 


\section{Abstract}

The RING finger type E3 ubiquitin ligase, Cbl-b, is abundantly expressed in bone marrow-derived mast cells (BMMCs) and functions as a potent negative regulator of signalling responses from the high affinity IgE receptor (FcERI). To determine the contribution of Cbl-b E3 ligase activity we generated knockin mice with a loss-offunction mutation in the RING finger domain. We find the mice to be healthy and, unlike equivalent c-Cbl RING finger mutant mice, produce homozygous offspring at the expected frequency. Comparative analyses of BMMCs from Cbl-b knockout and Cbl-b RING finger mutant mice revealed that both showed similarly enhanced FcERI signalling compared to wild-type cells for most parameters examined. A notable exception was a markedly higher level of activation of IKB kinase (IKK) in Cbl-b knockout BMMC compared to RING finger mutant derived cells. In addition BMMCs from the Cbl-b RING finger mutant did not retard FceRI internalization to the extent observed for knockout cells. Most striking however was the finding that RING finger mutant mast cells do not produce the very high levels of TNF- $\alpha$, IL-6 and MCP-1 evident in Cbl-b knockout cultures following FceRI activation. Thus the ability of Cbl-b to function as a negative regulator of FcERI signalling that promotes inflammatory cytokine production is largely independent of the RING finger domain. 


\section{Introduction}

The activation of mast cells and basophils by aggregated IgE is a key mechanism for inducing acute allergic reactions such as anaphylaxis and attacks of atopic asthma. These reactions are triggered by antigen-induced crosslinking of IgE bound to high affinity Fc receptors (FceRI) on the surface of mast cells and basophils. FceRI aggregation activates signalling cascades that promote many cellular effects including the release of preformed mediators of allergic reactions, the synthesis of pro-inflammatory lipid mediators and the synthesis and secretion of cytokines (reviewed in (Galli et al., 2005; Gilfillan and Tkaczyk, 2006; Kawakami and Galli, 2002)).

FceRI does not possess enzymatic activity but crosslinking with IgE and antigen does induce the activation of cytoplasmic protein tyrosine kinases of the Src, Syk and Tec families. The initiating kinase is the Src family member Lyn, which phosphorylates tyrosine residues in the immunoreceptor tyrosine-based activation motifs (ITAMs) of the $\beta$ and $\gamma$ subunits of FceRI that recruit Syk and additional Lyn molecules through interactions with their SH2 domains. Syk is then activated by Lyn, or newly activated Syk, enabling it to phosphorylate many downstream substrates. These Syk substrates, which include LAT (linker for activation of T cells), LAT2, SLP-76 (SH2-containing leukocyte protein of 76kDa) and Vav, activate pathways involving PLC $\gamma$, Rac, Ras, JNK and p38 (Hendricks-Taylor et al., 1997; Manetz et al., 2001; Saitoh et al., 2000; Volna et al., 2004; Zhu et al., 2004). The adaptor protein Gab2 (Grb2-associated-binder-2) has also been identified as an activator of the PI 3-kinase pathway in response to FceRI engagement (Gu et al., 2001). Interestingly Gab2 is phosphorylated by the Fyn kinase which is required for the activation of Akt (Parravicini et al., 2002). Together these signalling pathways contribute to induce the release of preformed histamine and the synthesis of multiple cytokines. Although many of these cytokines have protective roles in the clearance of bacteria and parasites, the overproduction of inflammatory cytokines such as 
TNF- $\alpha$, TGF $\beta$, IL-6 and monocyte chemotactic peptide-1 (MCP-1) can mediate damaging effects by promoting allergic and inflammatory reactions (reviewed in (Galli, 1993; Galli et al., 2005)).

It is clear that the activity of Syk is pivotal for triggering signalling responses in IgEactivated mast cells (Costello et al., 1996; Oliver et al., 1994; Zhang et al., 1996) thus providing a plausible target for suppressing pathogenic effects of allergic and inflammatory responses. The Cbl family of E3 ubiquitin ligases have emerged as candidates for inhibiting the activity of Syk by directing its ubiquitylation (Paolini et al., 2002; Rao et al., 2001). This E3 ubiquitin ligase activity is conferred by a RING finger domain that recruits ubiquitin conjugating enzymes (E2s) which then transfer associated ubiquitin molecules to the targeted substrate (Joazeiro et al., 1999; Levkowitz et al., 1999). Cbl proteins also possess numerous protein-protein interaction domains that serve to identify Cbl-specific substrates as well as recruiting additional signalling proteins to activated receptor complexes (Duan et al., 2004; Schmidt and Dikic, 2005; Thien and Langdon, 2005).

The mammalian Cbl family consists of three homologues, c-Cbl, Cbl-b and Cbl-3, and interestingly studies comparing bone marrow-derived mast cells (BMMC) from c-Cbl and Cbl-b knockout mice have revealed Cbl-b as the dominant protein that negatively regulates FcERI signalling (Gustin et al., 2006; Zhang et al., 2004). This finding was intriguing since Cbl-b and c-Cbl are very similar proteins and c-Cbl can very effectively ubiquitylate and negatively regulate Syk and FceRI in in vitro studies (Ota et al., 2000; Ota and Samelson, 1997; Paolini et al., 2002; Rao et al., 2001). However the marked enhancement of FceRI signalling by the loss of Cbl-b may simply be a consequence of the greater abundance of Cblb relative to c-Cbl protein in BMMCs (Gustin et al., 2006).

It is evident from over-expression studies of c-Cbl and Cbl-b in the basophilic cell line RBL-2H3 that the RING finger domain is necessary for the negative regulation of FceRI 
signalling (Ota and Samelson, 1997; Paolini et al., 2002; Qu et al., 2004). It was therefore surprising that a study comparing RBL-2H3 cells over-expressing membrane-targeted wildtype Cbl-b or a RING finger mutant (i.e. Cbl-b C373A) found that while the RING finger mutant was unable to suppress the activity of any signalling proteins examined it could still suppress the induction of TNF- $\alpha$ and IL-6 mRNA as effectively as wild-type Cbl-b (Qu et al., 2004). This suggested that Cbl-b regulates a yet to be identified pathway that is involved in the induction of inflammatory cytokines through a RING finger independent mechanism. This finding was of interest because our recent analysis of BMMCs from the Cbl-b knockout mouse showed that the most striking effect of the loss of Cbl-b was the marked enhancement in the induction of TNF- $\alpha$, IL-6 and MCP-1 in response to FceRI crosslinking (Gustin et al., 2006).

In this study we sought to clarify the role of the RING finger by generating a gene targeted mouse with the C373A loss-of-function mutation in the RING finger domain of Cblb and compared the effects of this mutation in BMMCs with that of the Cbl-b knockout. We find that BMMCs derived from the RING finger mutant are very similar to the Cbl-b knockout in the enhancement of many FceRI signalling responses, with a marked exception being the activation of I $\mathrm{kB}$ kinase (IKK) which is minimally affected by the loss of RING finger domain function. Significantly, we also find that the loss of a functional RING finger domain in Cbl-b does not markedly affect its ability to negatively regulate the production of TNF- $\alpha$, IL-6 and MCP-1. Thus Cbl-b’s negative regulation of FceRI signalling responsible for the production of inflammatory cytokines is largely independent of its E3 ligase activity and likely to involve suppression of NFkB signalling. 


\section{Materials and Methods}

\subsection{Generation of Cbl-b(C373A) knockin mice}

The generation of Cbl-b deficient mice has previously been described (Bachmaier et al., 2000). Normal control mice were obtained from our C57BL/6J x129Sv/J intercross breeding stocks. The Cbl-b(C373A) knockin mice were generated by Ozgene Pty Ltd (Australia). Briefly, a targeting vector introducing the Cys ( $\underline{\text { TGC) }}$-> Ala (GG) substitution at amino acid 373 (Fig.1B) was constructed using PCR fragments generated from 129Sv/J genomic DNA. Correctly targeted ES cell clones were identified by Southern blotting using 5' and 3' probes as indicated and used to establish 2 founder lines on a 129Sv/J x C57Bl/6 background. Excision of the loxP-flanked pGKNeo cassette was induced by mating with $\mathrm{C} 57 \mathrm{Bl} / 6 \mathrm{Cre}$ deleter transgenic mice. Mice were genotyped by PCR using a combination of primers $a-e$ (Fig. 1B) to detect wt and C373A targeted alleles and to confirm deletion of the pGKNeo cassette.

Primer sequences: $\quad a(w t), \quad$ 5'-GGCTCCACTTTTCAGCTCTGC-3'; $\quad a(C 373 A)$, 5’GGCTCCACTTTTCAGCTGGCG-3'; $b$, 5’-ATCTTCCCAGGAGCACGACG-3'; c, 5’TCTGAAGTTCGGTGGACC-3’; $d, \quad$ 5’- TGCTACTTCCATTTGTCACG-3’; $e, \quad$ 5’TGGCTCAGGAGAAGAGAAA-3’. Mouse experiments were performed in accordance with the Animal Ethics Committee at UWA (approval 03/100/275).

\subsection{Cell culture and antibodies}

Tissue culture flasks were obtained from Nunc (Roskilde, Denmark) and tissue culture media and supplements from ThermoTrace (Melbourne, Australia). Recombinant mouse IL-3 was a gift from Dr. Ian Young (JCSMR, Canberra) and DNP-BSA was obtained from Molecular Probes (Eugene, OR). Affinity purified mouse anti-DNP IgE was a gift from Drs. Janet 
Oliver and Bridget Wilson and has been previously described (Wilson et al., 2000). Mouse anti-Cbl-b (sc-8006) was purchased from Santa Cruz. Mouse anti-phosphotyrosine (4G10) was a gift from Dr. Brian Druker (Oregon Health Sciences University), and mouse anti-actin (A4700) was bought from Sigma. Rabbit anti-phospho-LAT (Y191), phospho-PKD (Ser916), phospho-PLC $\gamma 1$ (Y783), phospho-IKK- $\alpha / \beta($ Ser176/180), and IKK- $\alpha$ were from Cell Signalling Technology. FITC conjugated rat anti-mouse IgE and FITC-conjugated rat antimouse c-Kit (CD117) were purchased from BD Pharmingen.

\subsection{Bone marrow-derived mast cells from mice}

All experiments were performed in accordance with the Animal Ethics Committee at the UWA (approval 03/100/275). Bone marrow cells from 8-12 week old wild-type, Cbl-b knockout and Cbl-b(C373A) knockin mice (hereafter called BR mice for Cbl-b RING finger mutant) were cultured in RPMI 1640 supplemented with 10\% heat-inactivated FBS (Gibco/BRL), 50 $\mu \mathrm{M}$ 2-mercaptoethanol, $2 \mathrm{mM}$ L-glutamine and recombinant IL-3 at $37^{\circ} \mathrm{C}$ in 5\% $\mathrm{CO}_{2}$. Recombinant murine IL-3 was obtained from Dr Ian Young as supernatant from baculovirus infected Sf9 insect cells at a concentration of approximately 2 million units/ml. In all BMMC cultures we used the recommended concentration of 2,000 units/ml where 10 units is the amount of cytokine required to give 50\% proliferation of IL-3 dependent cells in a $1 \mathrm{ml}$ assay volume. The cells were split weekly into fresh media and tissue culture flasks at $5 \mathrm{x}$ $10^{5}$ cells $/ \mathrm{ml}$. By four weeks of culture more than $97 \%$ of the cells expressed uniformly high levels of FceRI and c-Kit.

\subsection{Mast cell stimulation and immunoblotting}

BMMCs at $2 \times 10^{6}$ cells/ml were stimulated by firstly priming with $1 \mu \mathrm{g} / \mathrm{ml}$ of IgE anti-DNP for 4-10 h in complete media followed by washes and resuspension in Tyrodes buffer (10mM 
HEPES pH 7.4, 130mM NaCl $2,1.4 \mathrm{mM} \mathrm{CaCl}_{2} 1 \mathrm{mM} \mathrm{MgCl}_{2}, 5 \mathrm{mM} \mathrm{KCl,} 5.6 \mathrm{mM}$ glucose and $0.1 \%$ BSA) at $10^{7}$ cells $/ \mathrm{ml}$. The antibody-primed BMMCs were activated by the addition of $10 \mathrm{ng} / \mathrm{ml}$ of DNP-BSA and incubation at 37C for $2-30 \mathrm{~min}$. The BMMCs were lysed in $n$ octyl- $\beta$-D-glucopyranoside/NP-40 lysis buffer (50 mM Tris-HCl, pH 7.4, $150 \mathrm{mM} \mathrm{NaCl,} 2$ mM EDTA, 1 mM sodium orthovanadate, $0.2 \%$ NP-40, 60mM $n$-octyl- $\beta$-D-glucopyranoside (Sigma), $10 \mu \mathrm{g} / \mathrm{ml}$ aprotinin, $1 \mathrm{mM}$ sodium orthovanadate, $10 \mathrm{mM} \mathrm{NaF}$, and $1 \mu \mathrm{g} / \mathrm{ml}$ each of chymostatin, leupeptin and pepstatin). After incubating for 15 min on ice, lysates were cleared by centrifugation at $3000 \mathrm{~g}$ for $8 \mathrm{~min}$ and prepared for SDS-PAGE. Lysates were analyzed by immunoblotting as previously described (Thien et al., 1999). Quantitation of protein bands was determined by densitometric analysis of scanned X-ray films (Canon CanoScan LiDE 20) using Kodak Molecular Imaging Software Version 4.0 (Eastman Kodak Company, New Haven CT).

\subsection{Cytokine assays}

BMMCs were primed with IgE anti-DNP as described above, washed and then cultured in complete media with $10 \mathrm{ng} / \mathrm{ml}$ DNP-BSA in 24 wells plates at $2 \times 10^{6}$ cells $/ \mathrm{ml}$ in $0.5 \mathrm{ml}$ for the indicated times. Supernatants were assayed for cytokine production using BD Cytometric Bead Array kits for mouse inflammatory cytokines (for detection of IL-12, IFN- $\gamma$, TNF- $\alpha$, MCP-1 and IL-6) or Bender Flowcytomix Simplex kits (for detection of IL-17, TNF- $\alpha$ and IL-6) according to the manufacturer's recommendations (BD Biosciences and Bender MedSystems, respectively). The beads were analyzed using a FACSCalibur flow cytometer and the data was analyzed using BD CBA software or Bender FlowCytomixPro 1.0 software. 


\subsection{Flow Cytometry}

Cell surface expression of FceRI and c-Kit were analyzed by a FACSCanto flow cytometer (BD Biosciences). Cells were incubated without IL-3 for $6 \mathrm{~h}$ before antibody staining. To determine FccRI levels the cells were primed with anti-DNP IgE as described above and IgE binding was detected with a FITC conjugated rat anti-mouse IgE (BD Pharmingen). Cells were collected using CellQuest software (BD) and further analyzed using FlowJo (Tree Star Inc). To assay for FceRI internalization $10^{6}$ BMMCs in $0.5 \mathrm{ml}$ were primed with IgE antiDNP in medium and stimulated with $10 \mathrm{ng} / \mathrm{ml}$ of DNP-BSA in Tyrodes buffer at $3 \mathbb{C}$ as described above. Receptor internalization was terminated over a range of times by adding 1 $\mathrm{ml}$ of ice-cold FACS buffer (PBS/1\% FCS with $0.5 \mathrm{mg} / \mathrm{ml} \mathrm{NaN}_{3}$ ). Surface FceRI levels at each time point were determined by staining with FITC conjugated rat anti-mouse IgE and flow cytometry.

\subsection{Pharmacological inhibitors}

BMMCs were primed with IgE anti-DNP as described above, washed and then cultured in complete media with or without inhibitors for PKC, PKC/PKD and PLC $\gamma$ for 30 min at $37^{\circ} \mathrm{C}$ before the addition of DNP-BSA. At various time points supernatants were harvested for the analysis of cytokine production. The inhibitors were purchased from Alexis Biochemicals and used as previously described, i.e. 400 nM for Gö6976, 200 nM for Ro-31-8220 and Gö6850, and $5 \mu \mathrm{M}$ for U-73122 (Dequiedt et al., 2005). Cycloheximide (Sigma-Aldrich) was used at $5 \mu \mathrm{g} / \mathrm{ml}$ for $1 \mathrm{~h}$ before addition of DNP-BSA. 


\section{Results}

3.1. A loss-of-function mutation in the Cbl-b RING domain does not affect the viability of mice

Mutant mice were generated with a Cys to Ala substitution at position 373 (i.e. C373A) located at the amino-terminus of the RING finger domain of Cbl-b (Fig. 1A and B, and Materials and Methods). Heterozygous C373A Cbl-b mice which we term +/BR (i.e. BR for B RING) were mated and litters genotyped by PCR analysis of genomic DNA (Fig. 1C). Immunoblotting of lysates from BMMC derived from wild-type and BR/BR mice with an anti-Cbl-b monoclonal antibody showed that Cbl-b protein levels are not affected by the C373A mutation (Fig. 1D).

Matings of +/BR mice gave the expected proportion of viable and healthy homozygous BR/BR offspring (i.e. of 274 mice produced $24 \%$ were genotyped as BR/BR, $52 \%+/ \mathrm{BR}$ and $24 \%+/+$ ). This is in marked contrast to the equivalent c-Cbl RING finger mutation where most homozygotes die in utero after day E14 and represent only 5\% of live births from heterozygous matings (Thien et al., 2005). Furthermore of these $~ 25 \%$ do not survive the first 24 hours after birth. This profound effect of the c-Cbl mutation on viability was considered to be due to the mutant protein acting as a dominant for Cbl-b since neither single knockout is lethal yet the double c-Cbl/Cbl-b knockout is lethal before day E10.5 (Liu and $\mathrm{Gu}, 2002$ ). The current finding that the Cbl-b RING finger mutation does not affect embryonic survival indicates that the dominant negative hypothesis as the explanation of embryonic death in c-Cbl RING finger mutant mice should be re-evaluated. Indeed the hypothesis that enhanced signalling by the c-Cbl RING finger protein itself causes embryonic death now appears more probable. In addition BR/BR mice do not have darker coat colors 
like the equivalent c-Cbl RING finger mutants (Thien et al., 2005), suggesting functional differences between the two homologues in regulating melanocyte signalling.

\subsection{Enhanced FceRI signalling in Cbl-b deficient and RING finger mutant mast cells}

It has previously been shown that Cbl-b is a key component in the negative regulation of signalling responses by FceRI-activated mast cells (Gustin et al., 2006; Qu et al., 2004; Zhang et al., 2004). To investigate whether a functional RING finger domain is required for this regulation we established BMMC cultures from wild-type, Cbl-b-/- and BR/BR mice and compared their signalling responses after priming with IgE anti-DNP and stimulating with DNP-BSA. Compared to BMMC lysates from wild-type mice, those from Cbl-b-/- and BR/BR mice showed higher levels of protein tyrosine phosphorylation at both the early time point of two minutes and the later time of 20 minutes following antigenic activation (Fig. 2A). The enhanced phosphotyrosine signal in Cbl-b-/- mast cells is consistent with previous studies (Gustin et al., 2006; Zhang et al., 2004) and this analysis has shown the RING finger domain to be an important component in regulating the strength of the FceRI signal that activates Lyn and Syk. We also consistently observed slightly higher levels of tyrosine phoshorylation in lysates from Cbl-b-/- cells compared to BR/BR cells, suggesting that domains outside the RING finger contribute to the negative regulation of FceRI signalling by Cbl-b.

The comparative strengths of FceRI signalling in the three BMMC lines were analysed in greater detail by immunoblotting with specific anti-phosphotyrosine antibodies to PLC $\gamma 1$ and LAT. Analysis of phospho-PLC $\gamma 1$ revealed higher levels of activation in both Cbl-b-/and BR/BR mast cells compared to wild-type cells and this signal was sustained over 20 minutes following the addition of antigen (Fig. 2B). This was confirmed by densitometry which showed a 50\% decrease in phospho-PLC $\gamma 1$ signal from 2 to 20 minutes in wild-type cells whereas in Cbl-b-/- and BR/BR mast cells this decreased by only $13 \%$ and $21 \%$ 
respectively. Analysis of LAT phosphorylation two minutes after antigenic stimulation revealed similar levels of activation between wild-type, BR/BR and Cbl-b-/- mast cells however by 20 minutes the levels in BR/BR and Cbl-b-/- mast cells were 10 and 20 fold higher respectively indicating a more sustained signal (Fig. 2B). Interestingly the antiphosphotyrosine immunoblot in Fig. 2A showed a comparatively greater phospho-LAT signal for Cbl-b-/- BMMCs at the 2 minute time point than with anti-phospho-LAT Y191 (Fig. 2B). Densitometry confirmed this observation by revealing a 2.7 and 2.2 fold greater signal for Cbl-b-/- than wild type and BR/BR mast cells respectively, whereas phospho-LAT Y191 only revealed a 1.2 fold increase (Fig. 2B). This difference is most likely due to enhanced tyrosine phosphorylation on sites other than Y191 and it would therefore be interesting to obtain antibodies that recognize the other phosphorylated tyrosines residues in LAT._ It should also be noted that Cbl-b-/- and BR/BR mast cells showed a higher level of constitutive phosphorylation of PLC $\gamma 1$ and LAT compared to wild-type cells, an observation consistent with the anti-phosphotyrosine immunoblot in Fig. 2A.

\subsection{Marked enhancement in the activation of IкB kinase in Cbl-b deficient mast cells}

We recently found that antigen-stimulated Cbl-b-/- mast cells exhibited a very high level of phosphorylation of IKK $\alpha / \beta$ (Gustin et al., 2006), the kinase complex responsible for the release of inactive NFאB into the nucleus. It was therefore important to establish whether the RING finger domain of Cbl-b is involved in the negative regulation of IKK $\alpha / \beta$ activation. Immunoblotting with antibodies directed against phosphorylated serines in the activation loop revealed that IKK $\alpha / \beta$ activation occurred more rapidly and was markedly higher in Cbl-b-/mast cells compared to either wild-type or BR/BR cells (Fig. 2C). This finding represents the most distinctive signalling difference we have observed between the two Cbl-b mutants to date. Fig. 2C also shows that Cbl-b has a profound effect in modulating IKK $\alpha / \beta$ activation 
since as shown here, and elsewhere, the time for maximal activation in Cbl-b knockout cells precedes that required in wild-type cells (Gustin et al., 2006). These findings implicate Cbl-b as key component in regulating the magnitude and kinetics of IKK $\alpha / \beta$ activation but suggest that the RING finger does not play a significant role in this regulation.

An upstream activator of IKK is the serine/threonine kinase protein kinase D (PKD, also known as PKC $\mu$ ) (Storz et al., 2004; Storz and Toker, 2003). PKD is also activated in mast cells in response to FceRI crosslinking and its catalytic activity can be efficiently monitored by the auto-phosphorylation of a C-terminal serine at residue 916 (Matthews et al., 2000). Immunoblotting with phospho-Ser916 antibodies showed a similar pattern to that observed for phospho-LAT where densitometry readings revealed an equivalently strong initial signal in wild-type cells at two minutes that decreased by $80 \%$ at 20 minutes (Fig. 2C). In contrast Cbl-b-/- and BR/BR mast cells increased their levels of phospho-PKD over this time period, most notably in Cbl-b-/- cells where this signal increased by $75 \%$. Thus ablation of Cbl-b or mutation of its RING finger domain also alters the kinetics of PKD activation.

\subsection{Loss of Cbl-b RING finger domain function has limited effects in enhancing inflammatory cytokine production}

We previously demonstrated that BMMCs from Cbl-b-/- mice show markedly enhanced production of the inflammatory cytokines TNF- $\alpha$, MCP-1, and IL-6 (Gustin et al., 2006). To test whether RING finger mutant mast cells show similar effects we primed wildtype, Cbl-b-/- and BR/BR mast cells with IgE anti-DNP and stimulated with DNP-BSA. As shown in Fig. 3, antigenic stimulation for 5 or 16 hours induced markedly higher levels of TNF- $\alpha$, MCP-1 and IL-6 from Cbl-b-/- mast cells compared to either BR/BR or wild-type cells. Although the BR/BR cells showed moderate increases of all three cytokines the levels did not approach the magnitude seen for Cbl-b-/- cells. We also examined IFN- $\gamma$, IL-10, IL- 
17, and IL-12p70 and, as previously found, the production of these cytokines by Cbl-b-/- mast cells was not enhanced ((Gustin et al., 2006) and data not shown).

We also tested whether the enhanced production of inflammatory cytokines by Cbl-b/- mast cells is regulated is dependent on de novo protein synthesis by pre-incubating and culturing in the presence of the protein synthesis inhibitor cycloheximide. This treatment was found to almost completely inhibit the production of MCP-1, TNF- $\alpha$, and IL-6 thus indicating that the high levels of these cytokines are due to the synthesis of new protein (data not shown). This finding is consistent with our previous study showing that the enhanced release of cytokines by Cbl-b-/- mast cells is not due to higher levels of preformed cytokines, since low and equal levels of intracellular TNF- $\alpha$ and IL-6 were observed in unstimulated Cbl-b-/and wild type mast cells (Gustin et al., 2006).

3.5. Loss of Cbl-b RING finger domain function does not result in a sustained block to FcERI internalization

Blocking the internalization and downregulation of antigen and growth factor receptors invariably results in a prolonged signalling response. We recently found that Cbl-b deficient BMMCs have a reduced ability to internalize FceRIs following antigen crosslinking suggesting that this perturbation may contribute to enhanced and sustained signalling (Gustin et al., 2006). Differential effects on receptor internalization might underlie the minimal impact of the RING finger mutant compared to the Cbl-b knockout on inflammatory cytokine production as seen in Fig. 3. We therefore compared the kinetics of antigen-induced FceRI internalization of wild-type, BR/BR and Cbl-b knockout mast cells in order to examine the role of the RING finger domain in this process. We found that the loss of cell-surface receptor over the initial 5 minutes after antigen crosslinking was retarded in BR/BR cells when compared to wild-type cells, but this perturbation was less pronounced than in Cbl-b 
knockout cells (Fig. 4). From this time on however BR/BR cells showed no apparent deficiency in their ability to progress with the internalization process, and by 20-30 minutes the BR/BR cells had similar receptor levels to wild-type cells as determined by geometric mean fluorescence (Fig. 4). In contrast, Cbl-b knockout BMMCs maintained a profound defect in FceRI internalization to the extent where they retained double the receptors on their surface compared to the two other cell lines. As previously found no additional internalization was detectable after 30 minutes of stimulation (Gustin et al., 2006).

\subsection{Changes in FceRI signalling intensities that affect inflammatory cytokine production are} not linked to altered FcERI internalization

The results shown in Fig. 4 suggest that a possible cause of the high levels of inflammatory cytokines produced by Cbl-b-/- mast cells could be the block to FceRI internalization which would result in a prolonged signal. It has been reported that lower concentrations of DNP can be optimal for triggering strong signalling responses, including the induction of some inflammatory cytokines, whereas higher concentrations can result in less intense responses and reduced production of some cytokines (Gonzales-Espinosa et al., 2003). We therefore utilized this approach to investigate whether high concentrations of DNP-BSA are more effective, and low concentrations less effective, in promoting FceRI internalization. If this were so it could provide a causal link to changes in cytokine production.

To test that high concentrations of DNP-BSA reduce inflammatory cytokine production we stimulated wild-type and Cbl-b-/- BMMCs with a range of concentrations of DNP-BSA and harvested the culture supernatants after 19 hours. In wild-type cells increasing the DNP-BSA concentrations from 10 to $100 \mathrm{ng} / \mathrm{ml}$ resulted in a $75 \%$ reduction in the production of TNF- $\alpha$ and IL-6, while treatment with $1000 \mathrm{ng} / \mathrm{ml}$ resulted in a 90\% reduction of both (Fig. 5A, top panels). The effect of increasing DNP-BSA concentrations on MCP-1 
production was less pronounced however a progressive inhibitory effect was clearly evident with a $50 \%$ reduction at the maximal concentration of $1000 \mathrm{ng} / \mathrm{ml}$ compared to $10 \mathrm{ng} / \mathrm{ml}$. The production of these cytokines by Cbl-b-/- mast cells was also inhibited by high antigen concentrations, but compared to wild-type cells the proportional decrease was less pronounced (Fig. 5A, lower panel). This effect may be attributed to the marked differences in the magnitude of responses between these two cell lines (n.b. the different scales of the Y axes in Fig. 5A), however it is clear that both are susceptible to changes in signal strength by varying the concentration of antigen.

We next investigated whether high concentrations of DNP-BSA are more effective in promoting FceRI internalization by stimulating wild-type and Cbl-b-/- BMMCs with a range of DNP-BSA-concentrations and measuring the extent of receptor internalization after 10 minutes. As illustrated in Fig. 5B FceRI internalization in both wild-type and Cbl-b-/- mast cells was not affected by increasing concentrations of DNP-BSA indicating that the reduced cytokine production is not caused by changes to FceRI internalization. These experiments therefore demonstrate that changes to signalling parameters that markedly affect cytokine production can occur with no corresponding alterations to FceRI internalization. This phenomenon raises the possibility that the massive increases we observe in inflammatory cytokine production by Cbl-b-/- mast cells may be not linked to the block in FceRI endocytosis.

\subsection{PKD is essential for the production of inflammatory cytokines}

The identification of PKD as a prominent target of sustained signalling in Cbl-b mutant mast cells (Fig. 2C) prompted us to test whether the activity of $\mathrm{PKD}$ is a requirement for the production of inflammatory cytokines. This analysis was undertaken by testing the 
effects of a range of inhibitors on the production of cytokines from Cbl-b-/- and BR/BR mast cells. The IgE-primed cells were pre-incubated with inhibitors for 30 minutes prior to the addition of DNP-BSA and the harvesting of culture media at 5 or 16 hours post stimulation (Fig. 6A and B respectively). Treatment with Gö6976, an inhibitor of classical PKCs and PKD, clearly prevented cytokine production as shown by the complete block in the production of TNF- $\alpha$ and IL-6, while MCP-1 production was reduced more than $70 \%$ (Fig. 6A and $\mathrm{B})$. In contrast two other inhibitors that are selective for classical and novel isoforms of PKC, but not PKD, (i.e. Ro-31-8220 and Gö6850) were ineffective at blocking FceRIinduced cytokine production (Fig. 6A and B). Not surprisingly, inhibition of PLC $\gamma 1$ with U73122 induced a total block in cytokine production. It was also apparent that the extent of inhibition by Gö6976 and U-73122 was comparable for Cbl-b-/- and BR/BR BMMCs. Overall these results indicate that the activities of PKD and PLC $\gamma 1$ are required for the induction of inflammatory cytokines in response to FceRI crosslinking, however since their activation is not markedly different between the Cbl-b mutants it is unlikely they are responsible for the excessive cytokine production by Cbl-b-/- mast cells. 


\section{Discussion}

The ability of the RING finger domain of Cbl proteins to associate with E2 ubiquitin conjugating enzymes is essential for their function as E3 ligases (Joazeiro et al., 1999; Levkowitz et al., 1999). Therefore studies of Cbl proteins with RING finger mutations that disrupt this association provide a means to identify $\mathrm{Cbl}$ substrates and characterize signalling pathways that are subsequently affected. This approach is well illustrated in a recent study comparing thymocytes from c-Cbl RING finger mutant mice with thymocytes from c-Cbl knockout mice where increased levels of antigen receptors and Src family kinases were found to be equivalently enhanced in both mutants (Thien et al., 2005). This analysis provided a clear demonstration that the absence of E3 ligase activity, and not the loss of other domains within c-Cbl, was the cause of these phenotypic changes. Here we carried out a similar comparative study by generating a knockin mouse with a loss-of-function mutation in the Cbl-b RING finger domain to determine whether enhanced FceRI signalling seen in Cbl-b deficient BMMCs can be attributed to the loss of E3 ligase activity. We find that following FceRI crosslinking, the activation of the protein tyrosine kinases Lyn and Syk, as measured by immunoblotting with anti-phosphotyrosine, anti-phospho-LAT and anti-phospho-PLC $\gamma 1$ antibodies, is enhanced and prolonged in mast cells from both Cbl-b mutants compared to wild-type cells (Fig. 2A and B). However in all experiments the Cbl-b deficient mast cells invariably displayed a greater level of activation than cells derived from the RING finger mutant. These experiments demonstrate that the RING finger domain of Cbl-b does play an important role in the negative regulation of FceRI-mediated activation of tyrosine kinase signalling but indicate that an additional domain or domains contribute to this regulatory function.

In our recent study comparing wild-type BMMCs with those derived from Cbl-b and c-Cbl knockout mice the most striking difference we found was the large induction of TNF- $\alpha$, 
IL-6 and MCP-1 in Cbl-b knockout cells in response to FceRI aggregation (Gustin et al., 2006). It was therefore of interest to determine whether this markedly deregulated production of inflammatory cytokines could be attributed to the loss of Cbl-b E3 ligase activity. We found that the RING finger domain had a limited role in negatively regulating the pathway that promotes the induction of these three cytokines (Fig. 3). This outcome was somewhat unexpected because we presumed that the E3 ubiquitin ligase activity of Cbl-b would invariably impact on all signalling responses that are linked to the activation of protein tyrosine kinases. Indeed, as mentioned above, we had previously shown that the equivalent loss-of-function mutation in c-Cbl recapitulated the enhanced tyrosine kinase signalling seen in the c-Cbl knockout, thus illustrating the importance of E3 ligase activity for c-Cbl function (Thien et al., 2005). As shown in this study this is clearly not the case for Cbl-b. These findings therefore provide functional evidence of a mammalian Cbl protein evolving from its invertebrate orthologue to develop a regulatory role that is independent of E3 ligase activity. This conclusion is supported by findings from the Komeda diabetes-prone (KPD) rat that expresses a Cbl-b protein with a large C-terminal truncation but it retains a functional RING finger domain (Yokoi et al., 2002). The study identified Cbl-b as a major susceptibility gene for insulin-dependent diabetes and the presence of this mutated form is associated with $\mathrm{T}$ cell activation, autoimmune disease and lymphocyte infiltration into pancreatic islets. These observations provide additional evidence that sequences C-terminal to the RING finger constitute an important component to Cbl-b’s ability to negatively regulate immune receptor signalling.

The observations that multiple domains are involved in regulating FceRI signalling are consistent with a study examining the effects of overexpressing c-Cbl constructs in RBL-2H3 basophilic leukemic cells (Ota and Samelson, 1997). It was found that neither the 70Z-Cbl mutant, which has a mutated RING finger domain, nor a C-terminally truncated Cbl-480 
construct, which loses SH3 and SH2-domain binding sites but retains an intact RING finger, could suppress the activation of Syk following FceRI crosslinking. In contrast a c-Cbl-655 construct that retains both the RING finger and proline-rich SH3 domain-binding region was as effective as wild-type c-Cbl in negatively regulating the activity of Syk. Cbl-b also shares many of the proline motifs that are present in c-Cbl between amino acid residues 481-655 and a study examining a C-terminally truncated Cbl-b protein equivalent to c-Cbl-480 was similarly found not to suppress Syk activation (Qu et al., 2005). Together these studies implicate the proline-rich region C-terminal to the RING finger domain as a contributing factor in the negative regulation of FceRI signalling by Cbl-b. Further characterization of this domain by determining the involvement of binding partners will thus be of interest for future studies.

We also found that antigen-induced internalization of FccRI is more severely retarded in Cbl-b knockout mast cells compared to RING finger mutant cells (Fig. 4). This is consistent with a recent study showing that the Cbl-interacting protein of $85 \mathrm{kDa}$ (CIN85) is involved in promoting antigen-dependent FceRI endocytosis in RBL-2H3 cells (Molfetta et al., 2005). CIN85 associates with a distinct proline-arginine motif conserved in the Cterminal regions of both Cbl-b and c-Cbl and this interaction enhances the internalization of numerous cell-surface receptors (reviewed by (Dikic, 2002)). Since this interaction would be retained in the Cbl-b RING finger domain mutant, but absent in the knockout, this may explain the distinct differences observed in FceRI internalization between the two mutants. Interestingly the CIN-85 binding site in Cbl-b is located C-terminal to the main proline-rich domain (Fig. 1A) so whether the loss of this site in Cbl-b would result in enhanced FceRI signalling and inflammatory cytokine production remains to be determined. However our findings in Fig. 5 demonstrating that changes to antigen-induced inflammatory cytokine production can occur in the absence of any effects on receptor internalization raises the 
possibility that loss of the CIN85 binding site may not be a significant factor contributing to the Cbl-b knockout phenotype.

Our analysis with chemical inhibitors clearly demonstrate the importance of PKD and PLC $\gamma 1$ activity for inflammatory cytokine production (Fig. 6) but the similarity of their activation in the two Cbl-b mutants suggest they are not responsible for the markedly greater cytokine production induced in Cbl-b knockout mast cells. Thus the signalling pathway so effectively regulated by Cbl-b to control the amplitude of inflammatory cytokine production is currently unknown. However it is intriguing that the most differentially enhanced signalling molecule found to date in Cbl-b knockout mast cells compared to the RING finger mutant and the c-Cbl knockout is IKK (Fig. 2C and (Gustin et al., 2006)). Interestingly this finding is in marked contrast to a study that examined overexpression of a membrane targeted Cbl-b (C373A) construct in RBL-2H3 cells where it was found that the RING finger mutant did not inhibit the activation IKK whereas membrane targeted wild-type Cbl-b did (Qu et al., 2004). This conflicting result is likely to be a consequence of the very different experimental systems for examing Cbl-b RING finger domain function.

To our knowledge there has only been one study of the role of IKK in mast cell signalling, and importantly this study found that IKK activity is required for the induction of TNF- $\alpha$ following FceRI engagement (Peng et al., 2005). It is well known that the NFkB pathway is required for the production of TNF- $\alpha$, IL-6 and MCP-1 so it appears that sequences in Cbl-b are involved in regulating the activity of this pathway. However the only published connection to date linking Cbl-b and the NFkB pathway is in T cells where Cbl-b overexpression was found to inhibit NFאB induction in a PI 3-kinase dependent manner (Herndon et al., 2005). It was hypothesized that this inhibitory effect was mediated by Cbl-bdirected ubiquitylation of p85, the regulatory subunit of PI 3-kinase. However since the effect we observe is largely independent of E3 ligase activity it is not likely to involve p85 
ubiquitylation. Furthermore an involvement with the PI 3-kinase pathway is unlikely as we have previously shown there is no enhancement in Akt activation in Cbl-b knockout mast cells compared to wild-type or c-Cbl-/- cells (Gustin et al., 2006).

Identifying the signalling molecule or molecules in the pathway between FceRI and IKK responsible for the marked enhancement of IKK activity when Cbl-b is ablated will be of fundamental importance to understanding this regulatory function of Cbl-b. This task is complicated by the large number of upstream MAP kinases that have been implicated in phosphorylating the activation loop in IKK $\alpha / \beta$, although only a few have been functionally verified by gene knockout experiments (reviewed by (Scheidereit, 2006)). These include NFאB-inducing kinase (NIK), MEKK3 and TGF $\beta$-activating kinase 1 (TAK1) and these represent suitable candidates for initial investigations. With IKK being the central point where most pathways that activate NFkB converge it will be of widespread significance to gain a better understanding of Cbl-b’s involvement in the negative regulation of its activity.

In conclusion this paper represent the first physiologically relevant study to examine the role of the Cbl-b RING finger domain in regulating FceRI signalling. Significantly we demonstrate that BMMCs derived from Cbl-b knockout and Cbl-b RING finger mutant mice show similarly enhanced FceRI signalling compared to wild-type cells for most parameters examined. A notable exception however is IKK which exhibits markedly higher activity in Cbl-b knockout derived mast cells. Strikingly, we also find that RING finger mutant mast cells do not produce the very high levels of TNF- $\alpha$, IL-6 and MCP-1 evident in Cbl-b knockout cultures following FceRI activation. Thus the ability of Cbl-b to function as a negative regulator of FceRI signalling that promotes inflammatory cytokine production is largely independent of the RING finger domain. 


\section{Acknowledgements}

We thank Josef Penninger for Cbl-b deficient mice, Ian Young for IL-3 and Brian Druker for 4G10 antibodies. We also thank Helen Moulder for animal care, Jay Steer for help with densitometry analysis and Kathy Heel for help with CBA flow cytometry carried out at The Centre for Microscopy and Microanalysis/Biomedical Image Facility. Funding was from NHMRC (Canberra) and MHRIF (W.A. Department of Health,). MPO was supported by a Fellowship from The Research Council of Norway and WYL by an NHMRC Fellowship.

\section{References}

Bachmaier K., Krawczyk C., Kozieradzki I., Kong Y.-Y., Sasaki T., Oliveira-dos-Santos A., Mariathasan S., Bouchard D., Wakeham A., Itie A., Le J., Ohashi P. S., Sarosi I., Nishina H., Lipkowitz S. and Penninger J. M. (2000). Negative regulation of lymphocyte activation and autoimmunity by the molecular adaptor Cbl-b. Nature 403, 211-216.

Costello P. S., Turner M., Walters A. E., Cunningham C. N., Bauer P. H., Downward J. and Tybulewicz V. L. (1996). Critical role for the tyrosine kinase Syk in signalling through the high affinity IgE receptor of mast cells. Oncogene 13, 2596-2605.

Dequiedt F., Van Lint J., Lecomte E., Van Duppen V., Seufferlein T., Vandenheede J. R., Wattiez R. and Kettmann R. (2005). Phosphorylation of histone deacetylase 7 by protein kinase D mediates T cell receptor-induced Nur77 expression and apoptosis. J. Exp. Med. 201, 793-804.

Dikic I. (2002). CIN85/CMS family of adaptor molecules. FEBS Lett. 529, 110-115.

Duan L., Reddi A. L., Ghosh A., Dimri M. and Band H. (2004). The Cbl family and other ubiquitin ligases: Destructive forces in control of antigen receptor signaling. Immunity 21, 7-17.

Galli S. J. (1993). New concepts about the mast cell. New Eng. J. Med. 328, 257-265.

Galli S. J., Kalesnikoff J., Grimbaldeston M. A., Piliponsky A. M., Williams C. M. M. and Tsai M. (2005). Mast cells as "tunable" effector and immunoregultory cells: Recent advances. Ann. Rev. Immunol. 23, 749-786.

Gilfillan A. M. and Tkaczyk C. (2006). Integrated signalling pathways for mast cell activation. Nature Rev. Immunol. 6, 218-230.

Gonzales-Espinosa C., Odom S., Olivera A., Hobson J. P., Eugena M., Martinez C., Oliveira-dosSantos A., Barra L., Spiegal S., Penninger J. M. and Rivera J. (2003). Preferential signaling and induction of allergy-promoting lymphokines upon weak stimulation of the high affinity IgE receptor on mast cells. J. Exp. Med. 197, 1453-1465.

Gu H., Salto K., Klaman L. D., Shen J., Fleming T., Wang Y. P., Pratt J. C., Lin G., Lim B., Kinet J.P. and Neel B. G. (2001). Essential role for Gab2 in the allergic response. Nature 412, 186190.

Gustin S. E., Thien C. B. F. and Langdon W. Y. (2006). Cbl-b is a negative regulator of inflammatory cytokines produced by IgE-activated mast cells. J. Immunol. 177, 5980-5989.

Hendricks-Taylor L. R., Motto D. G., Zhan J., Siraganian R. P. and Koretzky G. A. (1997). SLP-76 is a substrate of the high affinity IgE receptor-stimulated protein tyrosine kinases in rat basophilic leukemia cells. J. Biol. Chem. 272, 1363-1367.

Herndon T. M., Pirone D. M., Tsokos G. C. and Chen C. S. (2005). T cell-to-T cell clustering enhances NF- $\kappa$ B activity by a PI3K signal mediated by Cbl-b and Rho. Biochem. Biophys. Res. Comm. 332, 1133-1139.

Joazeiro C. A. P., Wing S. S., Huang H.-K., Leverson J. D., Hunter T. and Liu Y.-C. (1999). The tyrosine kinase negative regulator c-Cbl as a RING-type, E2-dependent ubiquitin-protein ligase. Science 286, 309-312. 
Kawakami T. and Galli S. J. (2002). Regulation of mast-cell and basophil function and survival by IgE. Nature Rev. Immunol. 2, 773-786.

Levkowitz G., Waterman H., Ettenberg S. A., Katz M., Lavi S., Iwai K., Reiss Y., Ciechanover A., Lipkowitz S. and Yarden Y. (1999). Ubiquitin ligase activity and tyrosine phosphorylation underlie suppression of growth factor signaling by c-Cbl/Sli-1. Mol. Cell 4, 1029-1040.

Liu Y. C. and Gu H. (2002). Cbl and Cbl-b in T-cell regulation. Trends Immunol. 23, 140-143.

Manetz T. S., Gonzalez-Espinosa C., Arudchandran R., Xirasagar V., Tybulewicz V. and Rivera J. (2001). Vav1 regulates phospholipase $\mathrm{C} \gamma$ and calcium responses in mast cells. Mol. Cell. Biol. 21, 3763-3774.

Matthews S. A., Rozengurt E. and Cantrell D. (2000). Protein Kinase D: A selective target for antigen receptors and a downstream target for Protein Kinase C in lymphocytes. J. Exp. Med. 191, 2075-2082.

Molfetta R., Belleudi F., Peruzzi G., Morrone S., Leone L., Dikic I., Piccoli M., Frati L., Torrisi M. R., Santoni A. and Paolini R. (2005). CIN85 regulates the ligand-dependent endocytosis of the IgE receptor: A new molecular mechansims to dampen mast cell function. J. Immunol. 175, 4208-4216.

Oliver J. M., Burg D. L., Wilson B. S., McLaughlin J. and Geahlen R. L. (1994). Inhibition of mast cell FceRI-mediated signaling and effector function by the Syk-selective inhibitor, piceatannol. J. Biol. Chem. 269, 15790-15796.

Ota S., Hazeki K., Rao N., Lupher M. L., Jr, Andoniou C. E., Druker B. and Band H. (2000). The RING finger domain of $\mathrm{Cbl}$ is essential for negative regulation of the Syk tyrosine kinase. J. Biol. Chem. 275, 414-422.

Ota Y. and Samelson L. E. (1997). The product of the proto-oncogene c-Cbl - a negative regulator of the Syk tyrosine kinase. Science 276, 418-420.

Paolini R., Molfetta R., Beitz L. O., Zhang J., Scharenberg A. M., Piccoli M., Frati L., Siraganian R. and Santoni A. (2002). Activation of Syk tyrosine kinase is required for c-Cbl-mediated ubiquitination of FceRI and Syk in RBL cells. J. Biol. Chem. 277, 36940-36947.

Parravicini V., Gadina M., Kovarova M., Odom S., Gonzalez-Espinosa C., Furumoto Y., Saitoh S., Samelson L. E., O'Shea J. J. and Rivera J. (2002). Fyn kinase initiates complementary signals required for IgE-dependent mast cell degranulation. Nature Immunol. 3, 741-748.

Peng Y., Power M. R., Li B. and Lin T. J. (2005). Inhibition of IKK down-regulates antigen + IgE-

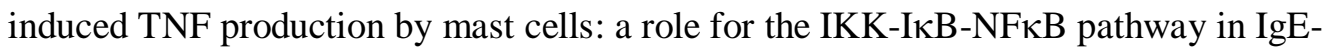
dependent mast cell activation. J. Leuk. Biol. 77, 975-983.

Qu X., Miah S. M. S., Hatani T., Okazaki M., Hori-Tamura N., Yamamura H., Hotta H. and Sada K. (2005). Selective inhibition of FceRI-mediated mast cell activation by a truncated variant of Cbl-b related to the rat model of Type I diabetes mellitus. J. Biochem. 137, 711-720.

Qu X., Sada K., Kyo S., Maeno K., Miah S. M. S. and Yamamura H. (2004). Negative regulation of FceRI-mediated mast cell activation by ubiquitin-protein ligase Cbl-b. Blood 103, 1779-1786.

Rao N., Ghosh A. K., Ota S., Zhou P., Reddi A. L., Hakezi K., Druker B. K., Wu J. and Band H. (2001). The non-receptor tyrosine kinase Syk is a target of Cbl-mediated ubiquitylation upon B-cell receptor stimulation. EMBO J. 20, 7085-7095.

Saitoh S., Arudchandran R., Manetz T. S., Zhang W., Sommers C. L., Love P. E., Rivera J. and Samelson L. E. (2000). LAT is essential for FceRI-mediated mast cell activation. Immunity $12,525-535$.

Scheidereit C. (2006). IkB kinase complexes; gateways to NF-kB activation and transcription. Oncogene 25, 6685-6705.

Schmidt M. H. H. and Dikic I. (2005). The Cbl interactome and its function. Nature Rev. Mol. Cell. Biol. 12, 907-919.

Storz P., Döppler H. and Toker A. (2004). Activation loop phosphorylation controls protein kinase Ddependent activation of nuclear factor $\kappa B$. Molecular Pharmacology 66, 870-879.

Storz P. and Toker A. (2003). Protein kinase D mediates a stress-induced NF- $\mathrm{KB}$ activation and survival pathway. EMBO J. 22, 109-120.

Thien C. B. F., Blystad F. D., Zhan Y., Lew A. M., Voigt V., Andoniou C. E. and Langdon W. Y. (2005). Loss of c-Cbl RING finger function results in high-intensity signaling and thymic 
deletion. EMBO J. 24, 3807-3819.

Thien C. B. F., Bowtell D. D. L. and Langdon W. Y. (1999). Perturbed regulation of ZAP-70 and sustained tyrosine phosphorylation of LAT and SLP-76 in c-Cbl-deficient thymocytes. J. Immunol. 162, 7133-7139.

Thien C. B. F. and Langdon W. Y. (2005). c-Cbl and Cbl-b ubiquitin ligases: substrate diversity and the negative regulation of signalling responses. Biochem. J. 391, 153-166.

Volna P., Lebduska P., Dráberová L., Símová S., Heneberg P., Boubelík M., Bugajev V., Malissen B., Wilson B. S., Horejsí V., Malissen M. and Dráber P. (2004). Negative regulation of mast cell signaling and function by the adaptor LAB/NTAL. J. Exp. Med. 200, 1001-1013.

Wilson B. S., Pfeiffer J. R. and Oliver J. M. (2000). Observing FceRI signaling from the inside of the mast cell membrane. J. Cell Biol. 149, 1131-1142.

Yokoi N., Komeda K., Wang H.-Y., Yano H., Kitada K., Saitoh Y., Seino Y., Yasuda K., Serikawa T. and Seino S. (2002). Cblb is a major susceptibility gene for rat type 1 diabetes mellitus. Nature Genet. 31, 391-394.

Zhang J., Berenstein E. H., Evans R. L. and Siraganian R. P. (1996). Transfection of Syk tyrosine kinase reconstitutes high affinity IgE receptor-mediated degranulation in a Syk-negative variant of rat basophilic leukemia RBL-2H3 cells. J. Exp. Med. 184, 71-79.

Zhang J., Chiang Y. J., Hodes R. J. and Siraganian R. P. (2004). Inactivation of c-Cbl or Cbl-b differentially affects signaling from the high affinity IgE receptor. J. Immunol. 173, 18111818.

Zhu M., Liu Y., Koonpaew S., Granillo O. and Zhang W. (2004). Positive and negative regulation of FceRI-mediated signaling by the adaptor protein LAB/NTAL. J. Exp. Med. 200, 991-1000. 


\section{Figure Legends}

\section{Fig. 1. Generation and identification of $\mathrm{Cbl-b}(\mathrm{C} 373 \mathrm{~A})$ mice.}

(A) Domains of Cbl-b showing the positions of the tyrosine kinase binding (TKB) domain, the RING finger (RF) domain with the location of the C373A mutation, the proline rich domain, the proline-arginine (PR) motif that binds CIN85/CMS SH3 domains and the ubiquitin-associated (UBA) domain. (B) Genomic organization of exons 5-9 of the mouse Cbl- $b$ gene showing the region targeted for homologous recombination to introduce the C373A mutation (asterisk). B, BamHI, S, StuI. The loxP-flanked pGKNeo cassette was removed by Cre-mediated excision in vivo, leaving a single loxP site (solid triangle). Mice were subsequently genotyped by PCR using a combination of primers a-e (Materials and Methods). (C) PCR genotyping of tail DNA from wild-type (+/+), heterozygous (+/BR) or homozygous Cbl-b (C373A) mutant (BR/BR) mice using primer $b$ with either primer $a(w t)$ (top panel) or $a(C 373 A)$ (bottom panel) to detect the wild-type and C373A targeted alleles

respectively. (D) Anti-Cbl-b immunoblot of total cell lysates from BMMC derived from wild-type (+/+), Cbl-b knockout (b-/-) and homozygous Cbl-b (C373A) RING finger mutant mice (BR). The amount of lysate loaded in each track was examined by immunoblotting with an anti-actin monoclonal antibody.

Fig. 2. Enhanced FceRI signalling in mast cells from the Cbl-b knockout and RING finger mutant mice.

IgE-primed wild-type (+/+), Cbl-b-/- (b-/-), and Cbl-b RING finger mutant (BR) mast cells were left unstimulated or activated with $10 \mathrm{ng} / \mathrm{ml}$ of DNP-BSA for 2 or 20 minutes. Cell lysates were analyzed by immunoblotting as follows. (A) Immunoblot probed with 4G10 anti-phosphotyrosine (pTyr) antibodies. Molecular weight markers are indicated in kDa as are the mobilities of the Lyn and Syk kinases and substrates SLP-76 and LAT. (B) 
Immunoblots were probed with phospho-specific antibodies to PLC $\gamma 1(Y 783)$ and LAT(Y191) and (C) IKK $\alpha / \beta(S 176 / 180)$ and $\mathrm{PKD}(\mathrm{S} 916)$. The numbers below the phospho-specific immunoblots represent densitometry readings of each band expressed relative to the signal obtained at the two-minute time point from $+/+$ lysates. All values have been adjusted for loading variations as determined by reprobing the immunoblots with the relevant proteinspecific or anti-actin antibodies. Each immunoblot is representative of three experiments from two independently derived sets of BMMCs established from +/+, b-/- and BR mice.

Fig. 3. Cbl-b-/- BMMCs show markedly enhanced production of the inflammatory cytokines TNF- $\alpha$, MCP-1 and IL-6.

IgE-primed BMMCs from wild-type (+/+), Cbl-b-/- (b-/-) and Cbl-b RING finger mutant (BR) mice were left unstimulated or stimulated with $10 \mathrm{ng} / \mathrm{ml}$ of DNP-BSA for 5 hours (A) or 16 hours (B). The concentrations of inflammatory cytokines in the culture supernatants were determined by Cytometric Bead Array assays and the results are from two independently established sets of BMMC cultures. The cells were examined at 8 weeks (A) and 7 weeks (B) after establishment. Please note that the Cbl-b+/+ culture in B produced $33 \mathrm{pg} / \mathrm{ml}$ of TNF$\alpha$ and $83 \mathrm{pg} / \mathrm{ml}$ of IL-6. These values are not evident on the graph because of the large scale required for the $\mathrm{Y}$ axis. The results are representative of experiments carried out two and three times on two independently derived BMMCs established from +/+, b-/- and BR mice.

Fig. 4. Antigen-induced FceRI internalization is retarded in Cbl-b-/- BMMCs.

Cells from wild-type (+/+), Cbl-b-/- (b-/-) and Cbl-b RING finger mutant (BR) mice were primed with IgE anti-DNP and crosslinked with DNP-BSA at $37^{\circ} \mathrm{C}$ for the indicated times in minutes (m). Receptor internalization was terminated at each time point by the addition of ice-cold FACS buffer and surface levels of FceRI were determined by staining with FITC conjugated rat anti-mouse IgE and analyzed flow cytometry. The numbers in each panel indicate the geometric mean fluorescence of each sample. The results are representative of 
experiments carried out twice on two independently established BMMCs from +/+, b-/- and BR mice.

Fig. 5. DNP dose response of IgE-mediated cytokine production and FceRI internalization.

(A) IgE-primed BMMCs from wild-type (+/+) and Cbl-b-/- (b-/-) mice were left unstimulated or stimulated with a range of concentrations of DNP-BSA for 19 hours. The concentrations of inflammatory cytokines in the culture supernatants were determined by Cytometric Bead Array assays. Please note that scales of the $\mathrm{Y}$ axes are markedly different between the $+/+$ and b-/- cultures. (B) BMMCs from wild-type (+/+) and Cbl-b-/- (b-/-) mice were primed with IgE anti-DNP and crosslinked with increasing concentrations (10-1000 ng/ml) of DNP$\mathrm{BSA}$ at $37^{\circ} \mathrm{C}$ for $10 \mathrm{~min}$. Receptor internalization was terminated by the addition of ice-cold FACS buffer and surface levels of FceRI were determined by staining with FITC conjugated rat anti-mouse IgE and analyzed by flow cytometry. The dashed line represents staining by anti-IgE-FITC on mast cells that were not primed with IgE. The results are representative of data from two independently established sets of BMMC cultures from experiments carried out once and twice on each culture set.

Fig. 6. PKD activity is required for the production of inflammatory cytokines TNF- $\alpha$, MCP-1 and IL-6.

IgE-primed BMMCs from Cbl-b-/- (b-/-) and Cbl-b RING finger mutant (BR) mice were left untreated or incubated for 30min with an inhibitor for classical PKC and PKD (Gö6976), inhibitors for classical and novel PKC isoforms (Ro-31-8220 and Gö6850), or an inhibitor for phospholipase-C (U73122). The cells were thereafter incubated with or without $10 \mathrm{ng} / \mathrm{ml}$ of DNP-BSA for 5 hours (A) or 16 hours (B). The concentrations of inflammatory cytokines in the culture supernatants were determined by Cytometric Bead Array assays. The results are representative of three separate experiments from a single set of BMMCs. 